\title{
STELLAR EVOLUTION IN REAL TIME: CURRENT AND FUTURE EXPECTATIONS
}

\author{
YOJI KONDO \\ NASA Goddard Space Flight Center
}

\section{Introduction}

Directly measuring stellar evolution may be accomplished in at least two distinctly different ways. The first approach will be observing it in real time as the phenomenon is taking place; the examples will include explosive events in recurrent novae, stel- lar oscillations in Cepheid and RR Lyrae variables, and mass flow in and out of interacting binaries. The second approach will be to observe a number of stars that are believed to belong to the same class at different stages of their evolutionary paths.

In this talk, I will focus on the first method of direct observations. The most direct method will probably be to observe images of the events that we wish to investigate. The same astronomical phenomena can and should also be studied using high-time and spectroscopic resolution data; however, this is already being done with increasingly better spectral-and-temporal resolutions and will probably be reported elsewhere in this Joint Discussion. It is also noted that over the past two decades multiwavelength observations, using various telescopes on the ground and in space, have come to play a crucial role in astronomy, although I am not addressing this subject in this paper. I will talk primarily about imaging using interferometry that will soon become possible due to the advances in technology.

\section{Observing Stellar Evolution in Real Time with Interferometry}

\subsection{GROUND-BASED TELESCOPES}

1) An array of five 1-meter telescopes at Mount Wilson with a maximum separation of 150 meters is being constructed at Mount Wilson under the leadership of Harold McAlister of the Center for High Angular Resolution Astronomy (CHARA) at Georgia State Uni- versity. Adaptive optics will be used for the mirrors. [As a point of interest, the 100-inch telescope at Mt. Wilson is presently used successfully with adaptive optics]. An approxi- mate resolution of $10 \times 10^{-9}$ radian will have an image resolution of $10 \times 10^{11} \mathrm{~cm}$ at a distance of 10 light years. The base can be ex- tended to a 1,000 meters if desired. The first light is expected before the end of this century, which will come in three years.

2) Interferometry technology is also being applied to the Keck 10-meter telescope at Mauna Kea in Hawaii and to the 5-meter reflector at Palomar Mountain. As presently envisaged, the baselines for those two projects will be shorter than that for the CHARA array; their mirrors will however have greater apertures, which should be an asset in achieving high angular resolution. 


\subsection{TELESCOPES IN FREE ORBIT}

There currently are three projects under active planning at NASA.

1) Space Interferometry Mission (SIM) with five $40-\mathrm{cm}$ class telescopes on a 10 -meter beam (baseline) is being planned for early next century.

2) Deep Space Three Mission, which involves an array of free-orbiting telescopes, will test the technology crucial to future interferometry in space; for this technology to work, we will need to know the positions of each telescope to within a few percent of the wavelength at which observations are being made. This technology will be crucial in realizing astronomically-long baseline interferometry in the future; attainable resolution will become virtually unlimited with it.

3) Terrestrial Planet Finder (TPF) Mission on a 50-to-75 meter platform will be sent to beyond Jupiter's orbit. This is to avoid the background noise from the zodiacal light within our solar system. [Not much can be done about any zodiacal light background at the other end.]

4) We should perhaps mention that the New Generation Space Telescope (NGST) with an aperture of 8 meters will improve the resolution currently attained with the 2.4-meter Hubble Space Telescope by more than a factor of 3 .

\subsection{INTERFEROMETRIC TELESCOPES ON THE MOON}

Even before the technology being tested in Deep Space Three (B: 2) becomes available, we can consider the construction of an array of interferometric telescopes on the Moon; our natural satellite is essentially an immense optical bench in vacuum. Using innovative technologies (Chen, Kondo \& Oliversen), the cost of placing a one-meter class telescope on the lunar surface can now be brought down to the range of NASA's MIDEX projects, i.e., to the several times $\$ 10 \mathrm{M}$ range. Once reusable launch vehicles (RLVs) reduce the cost of placing payloads in low Earth orbit by a factor of ten to a hundred, the cost of getting telescopes to the lunar surface can decline correspondingly, making the concept of interferometry from the Moon even more economical.

The natural curvature of the Moon will give us a baseline of about a kilometer. However, this baseline can be extended significantly using various methods, including the use of struts, lunar crater limbs, and optical relays. In this way, we can eventually attain a resolution of $10 \times 10^{-12}$ radian or higher, which should give $1 \mathrm{~s}$ a $10 \times 10^{+9} \mathrm{~cm}$ resolution or better for objects at a distance of some 1,000 light years. (As a point of comparison, the solar diameter is greater than $10 \times 10^{+11}$ cm.)

See: Chen, P., Kondo, Y. \& Oliversen, R, in JD-22 "Astronomy from the Moon", Highlights of Astronomy for the 1997 IAU General Assembly. 